\title{
Feature Extraction of Precession Targets Based on Multi-aspect Range-Profile Sequence
}

\author{
Dan Xu a , Bo Tian ${ }^{b}$, Cunqian Feng ${ }^{c}$, Shuang Zhao ${ }^{d}$, Yizhe Wang ${ }^{e}$ \\ Air and Missile Defense College, Air Force Engineering University, Xi'an, 710051, China; \\ axudanlw@163.com, btianbo0216@163.com, cfengcunqian@sina.com, dzhaoshuanglw@163.com, \\ ewangyizhe813@163.com.
}

\begin{abstract}
Parameters extraction of precession targets are important information source for ballistic target recognition. Aiming at the limited ability of extracting targets parameters with single radar, an algorithm based on uniting the multi-aspect observation information is proposed to extract the precession features of ballistic targets in this paper. Firstly, cone-shaped precession model and wideband radar signal model are established and different-aspect conic node scattering point is matched and identified based on analyzing the relationship of each scattering point's micro-distance variation in detail. Then, utilizing the variation information of target's projected length obtained from range-profile sequence to estimate precession parameters, which can obtain estimation of precession parameters and target structure parameters without any prior information. Lastly, Simulation results prove that the proposed method can realize the extraction of high accuracy parameters.
\end{abstract}

Keywords: Range-profile sequence; Precession; Scattering point matching; Multi-aspect.

\section{Introduction}

As the inherent feature of moving target, micro-motion feature can be used for describing the weak kinematic features, or even the kinematic features and motion form of the structure on the target. In recent years, ballistic target micro-motion feature is one of the crucial features used for radar target recognition [1-2]. However, single radar shows the deficiency of sensitive to target attitude and extracting micro-motion parameters inaccurately in ballistic target recognition. Netted radar can obtain micro-motion from different aspect, so it can overcome the deficiency of sensitive to target attitude and improve the accuracy of extracting micro-motion parameters.

Compared with narrowband radar, wideband radar can obtain elaborate target configuration parameters due to high bandwidth. LEI et al. deduced scattering points' radial distance mathematic model and obtain the linear sum signal of scatters' radial distance. Precession parameters can be extracted by the sum signal's spectral characteristics [3]. AI et al. indicated that the sequence of HRRP lengths can be approximated to a sinusoid function. Precession angle and real target length can be estimated after uniting multi-aspect [4]. YAO et al. analyzed the HRRPs of the rotary symmetrical cone-cylinder target in four attitude angle regions respectively. The precession and structure parameters are estimated based on the relationship among the extreme value of three scattering points' relative position in the HRRPs [5]. HE utilized the variation information of target's projected length which obtained from HRRP sequences to structure system of linear equations. Precession parameters and target structure parameters are estimated based on system of linear equations [6].

On the basic of the reference [6], feature extraction of the cone-shaped target in the condition of three scattering points being visible based on multi-aspect range profile sequence. Scattering point is matched by analyzing the relationship of each scattering point's micro-distance variation. On the basic of scattering point being matched, regarding the conic node observed information of two radars as one solving unit and parameters from each unit are solved. Then, other parameters can be solved by micro-motion information of the basilar cone. Simulation results are given for validating the proposed algorithms. 


\section{Model building}

\subsection{Cone-shaped precession model}

The sketch map of netted radar is shown in fig. 1 and the sketch map of cone precession model is shown in fig.2. $O_{0} X^{\prime} Y^{\prime} Z^{\prime}$ Is the global coordinate system which includes all the radars? $O X Y Z$ Is the reference coordinates system. $O$ Is the intersection of coning axis and target's symmetry axis? Coning axis is the $Z$ axis. $Y O Z$ Is the level which includes the symmetry axis and $Z$ axis at the beginning time? The $X$ axis's direction is obtained according to the right hand spiral standard. The coning angular velocity is $\omega_{c}$. The precession angle is $\theta$. The semi-cone angle is $\varepsilon$. The height of cone is $h$. The radius of cone is $r$. The distance from the conic node to $O$ is $h_{1}$. The distance from cone of bottom center to $O$ be $h_{2}$. The cone target initial azimuth angle is $\varphi_{0}$. The azimuth angle of LOS in the reference coordinates system is $\varphi_{i}$. The angle between LOS and $Z$ axis is $\alpha_{i} . p$ And $q$ are the intersection of LOS and the trajectory target bottom edge structure. The angle between LOS and line of symmetry is $\beta$. The distance from $O$ to radar is $R_{0}$ and meets far-field condition.

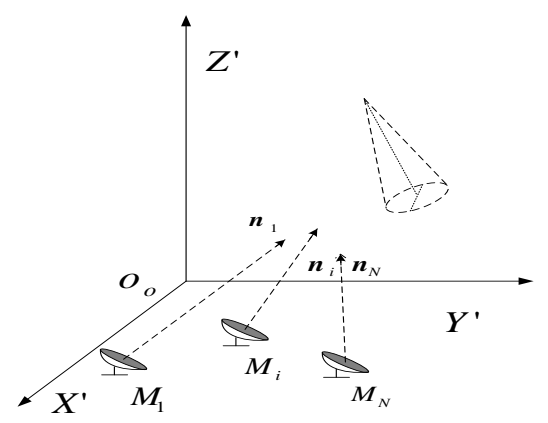

Fig. 1 The sketch map of netted radar

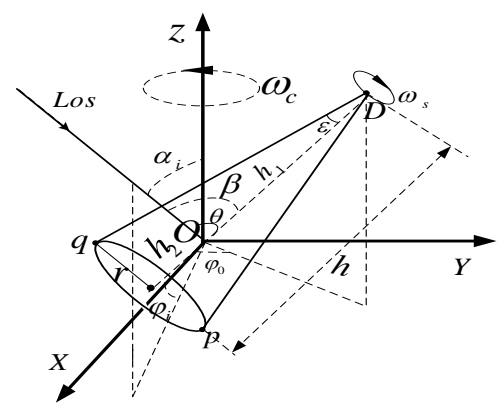

Fig. 2 The sketch map of cone precession model

For rotationally symmetric target, its high frequency scattering characteristic is mainly determined by the conic node scattering point and the basilar cone scattering points on the basic of optical zone and taking no account of shaded effectiveness ${ }^{[2]}$. For single radar, when $\beta \in(0, \varepsilon) \bigcup(\pi / 2, \pi-\varepsilon)^{[7]}$, three scattering points can be observed at the same time. The paper mainly analyzes precession when $\beta \in(\pi / 2, \pi-\varepsilon)$ because of $\varepsilon$ smaller ${ }^{[7]}$. Through geometrical deduction, angle of radar sight $(\beta)$ satisfied expression as follows:

$\cos \beta(t)=\cos \theta \cos \alpha_{i}+\sin \theta \sin \alpha_{i} \sin \left(\omega_{c} t-\varphi_{0}\right)$

So scattering points radial distane expression as follows:

$\left\{\begin{array}{l}R_{D}=R_{0}+h_{1} \cos \beta(t) \\ R_{p}=R_{0}-h_{2} \cos \beta(t)+r \sin \beta(t) \\ R_{q}=R_{0}-h_{2} \cos \beta(t)-r \sin \beta(t)\end{array}\right.$

\subsection{Wideband signal model}

Assume that wideband radar transmits LFM signal:

$$
\begin{aligned}
& s_{t}\left(t_{m}, t_{k}\right)=\operatorname{rect}\left(\frac{t_{k}}{T_{p}}\right) \cdot \exp \left(j 2 \pi\left(f_{c} t_{k}+\frac{1}{2} \gamma t_{k}^{2}\right)\right) \\
& \operatorname{rect}\left(\frac{t_{k}}{T_{p}}\right)= \begin{cases}1, & -T_{p} / 2 \leq t_{k} \leq T_{p} / 2 \\
0, & \text { other }\end{cases}
\end{aligned}
$$

In formula (1) and (2), $T_{p}$ is the pulse width, $f_{c}$ is the signal carrier frequency, $\gamma$ is the adjustable frequency, $t_{k}=t-m T_{r}$ is fast time, $t_{m}=m T_{r}$ is slow time, $T_{r}$ and is the pulse repeated frequency. $m=0,1, \cdots, M-1$ Is the number of pulse sequence? Assume that translation of cone is compensatory, $R_{r e f}=R_{0}$ and $R_{\Delta l}\left(t_{m}\right)=\mathrm{R}_{l}\left(t_{m}\right)-R_{r e f}$. Taking the $O$ echo signal as reference signal, after stretch, RVP and enveloping diagonal processing, the expression of scattering points is listed as follows: 


$$
S_{r}\left(f, t_{m}\right)=\sum_{l=1}^{L} \sigma_{l} T_{p} \operatorname{sinc}\left(T_{p}\left(f+\frac{2 \gamma}{c} \cdot R_{\Delta l}\left(t_{m}\right)\right)\right) \exp \left\{-j \frac{4 \pi f_{c}}{c} R_{\Delta l}\left(t_{m}\right)\right\}
$$

In formula (5), $L$ is the number of scattering, $\sigma_{l}$ is the scattering coefficient of $l$.Range as the peak expression:

$$
f_{l}=-\frac{2 \gamma}{c} \cdot R_{\Delta l}\left(t_{m}\right)
$$

The formula (6) on both sides at the same time multiplied by $-c /(2 \gamma)$ can keep the position of scattering point's range as the peak and micro-distance. Combining with the formula (2), the expression of scattering point micro-range is listed as follows:

$$
\left\{\begin{array}{l}
R_{D}=h_{1} \cos \beta(t) \\
R_{p}=-h_{2} \cos \beta(t)+r \sin \beta(t) \\
R_{q}=-h_{2} \cos \beta(t)-r \sin \beta(t)
\end{array}\right.
$$

Combining with formula (1) and (7), we can conclude that the expression of scattering point $D$ 's micro-range is simpler than the expression of scattering point $p$ 's and $q$ 's micro-range. The change rule of scattering point $D$ 's micro-range meets sinusoidal. But scattering point $p$ 's and $q$ 's micro-range meets a sum of sinusoidal component and non-sinusoidal component. So, in this paper, we take the simpler scattering point $D$ into account to analyze precession and extract parameters.

\section{Scattering point matching}

Generally, the location of radar is different in netted radar. Different radar location has different observation angle, it can lead to the projective position of target's scattering point on the radar direction different at the same time. Accordingly, different radar observing the same scattering point at the same time will obtain different range-profile sequence. Therefore, utilizing the netted radar multi-aspect observation information needs to know range-profile the corresponding scattering point. Through observing the expression (7), we can get that:

$$
\frac{-2 h_{2}}{h_{1}}=\frac{R_{p}+R_{q}}{R_{D}}
$$

In formula (8), $h_{1}$ and $h_{2}$ are target's structure parameters and bear no relation to radar observation. So the ratio of two basilar scattering point micro-range's sum and conic node scattering point is a constant value at the same time, but the other ratio units are not constant value. So scattering point $D$ is matched. However, scattering point $p$ and $q$ are sliding and reciprocally transform. They are difficult to be matched. Therefore, don't separate scattering point $p$ and $q$ to analyze precession.

\section{Precession parameters and structure parameters solving}

In formula (1), precession angle $\theta$ is coupled with the radar observation angle $\alpha_{i}$. It's difficult for single radar to obtain parameters. Two radars' observation information can solve parameters at least. Extracting range-profile sequence of conic node scattering point from the $i$ radar, we assume that mean value is $l_{C i}$ and amplitude is $l_{A i}$. The formula (9) can be obtained by substituting formula (1) into formula (7).

$$
\left\{\begin{array}{l}
R_{A}=h_{1} \cos \beta(t)=h_{1} \cos \theta \cos \alpha_{i}+h_{1} \sin \theta \sin \alpha_{i} \sin \left(\omega_{c} t+\varphi_{i}\right) \\
l_{C i}=h_{1} \cos \theta \cos \alpha_{i} \\
l_{A i}=h_{1} \sin \theta \sin \alpha_{i}
\end{array}\right.
$$

Utilizing range-profile sequence of conic node scattering point from the $i$ radar and $j$ radar, parameters being solved are listed as follows:

$$
\theta=a \tan \sqrt{\frac{l_{A i}^{2}-l_{A j}^{2}}{l_{C j}^{2}-l_{C i}^{2}}}
$$




$$
\begin{gathered}
h_{1}=\sqrt{\left(\frac{l_{C i}^{2}}{\cos ^{2} \theta}+\frac{l_{A i}^{2}}{\sin ^{2} \theta}\right)} \\
\left\{\begin{array}{l}
\alpha_{i}=a \cos \left(\frac{l_{C i}}{h_{1} \cos \theta}\right) \\
\alpha_{j}=a \cos \left(\frac{l_{C j}}{h_{1} \cos \theta}\right)
\end{array}\right.
\end{gathered}
$$

The formula (13) can be obtained by the formula (7):

$$
\left|R_{q}-R_{p}\right|=2 \cdot|r \sin \beta(t)|
$$

$\cos \beta(t)$ Can be solved by uniting the formula (9) and the formula (11), so $r$ can be solved by the formula (13). $h_{2}$ Can be solved by substituting formula (1) into formula (7). Cone palstance $\omega_{c}$ can be solved by the reference [8].

According to all the analysis above, the steps to extract feature of precession targets based on multi-aspect range-profile sequence are:

Step 1: Setup distributed radar network, assuming radars are all synchronized and estimating LFM signal, and there are two or more radars in the network.

Step 2: Taking any radar from the netted radar to analyze the cone-shaped precession, and solving the expression of micro-distance. The value of scattering points' range-profile sequence at a certain time can be extracted by the ESPRIT ${ }^{[9]}$ algorithm.

Step 3: The range-profile sequence curve of scattering point $D$ is matched by the value of scattering points' range-profile sequence.

Step 4: Mean value and amplitude can be extracted by utilizing the ESPRIT ${ }^{[9]}$ algorithm based on two radars' range-profile sequence curve of scattering point $D$. The parameters can be estimated by formula (10) to (13).

By taking steps all above, many features of cone target can be extracted. Differ from the single based radar, radar network achieved to extract the features more precisely.

\section{Simulations}

Simulation dada: Assuming that target is symmetric cone, there are 3 radars in netted radar. Target parameter data: $h=2.5 \mathrm{~m}, h_{1}=2 \mathrm{~m}, h_{2}=0.5 \mathrm{~m}, r=0.5 \mathrm{~m}, \theta=10^{\circ}, \varphi_{0}=45^{\circ}, \omega_{c}=8 \pi \mathrm{rad} / \mathrm{s}$. The angles between LOS and $Z$ axis are $\alpha_{1}=100^{\circ}, \alpha_{2}=115^{\circ}$ and $\alpha_{2}=130^{\circ}$. The line frequency modulation signal radar's carry frequency is $10 \mathrm{GHz}$. The pulse repeated frequency is $1 \mathrm{KHz}$, the bandwidth is $4 \mathrm{GHz}$, and echo signal-to-noise ratio is $10 \mathrm{~dB}$. The observation time is 1 second.

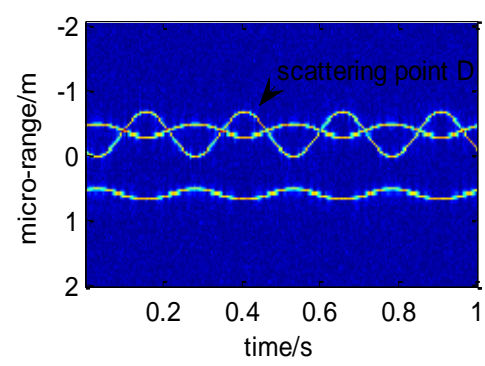

(a) radar 1

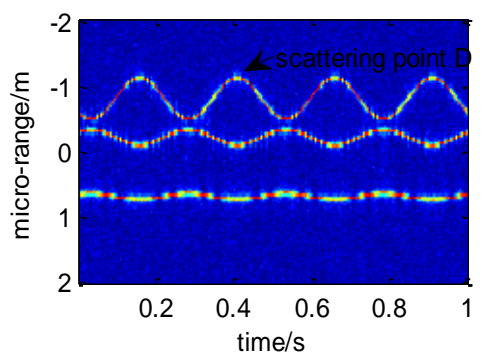

(b) radar 2

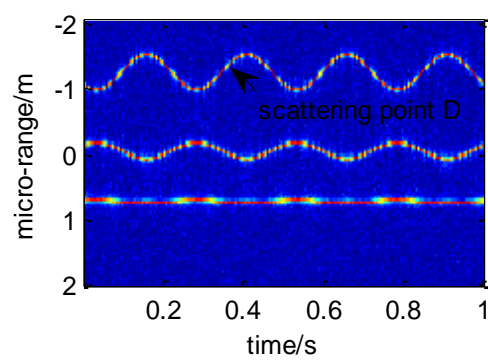

(c) radar 3

Fig. 3 The range time-profile sequences of the netted radar's echo

Fig. 3 is the range time-profile sequences of the netted radar's echo. There are three curves in the picture and one curve meets sinusoidal. Fig4 is the range profile sequence of the netted radar at certain time. The data listed in Table 1 is extracted by utilizing the ESPRIT ${ }^{[9]}$ algorithm from Fig. 4 According to the third quarter, the scattering point $D$ is matched based on the data listed in Table 1 
So we know that $s 1\left(t \mid \alpha_{1}\right)$ is scattering point $D$ as well as the value of $\left|s 2\left(t \mid \alpha_{1}\right)-s 3\left(t \mid \alpha_{1}\right)\right|=\left|R_{p}\left(t \mid \alpha_{1}\right)-R_{q}\left(t \mid \alpha_{1}\right)\right|$ providing the basis of solving $r$. Radar 1 and radar 2 are regarded as a solving unit 1 , radar 1 and radar 3 are regarded as a solving unit 2 , radar 2 and radar 3 are regarded as a solving unit 3 . The precession angle $\theta_{1}$ is solved by solving unit 1 . The precession angle $\theta_{2}$ is solved by solving unit 2 . The precession angle $\theta_{3}$ is solved by solving unit 3 . The precession angle $\theta$ is solved by the average of $\theta_{1}, \theta_{2}$ and $\theta_{3}$.The precession parameters and structure parameters are solved and listed in Table 2. And the error is within the allowable range.

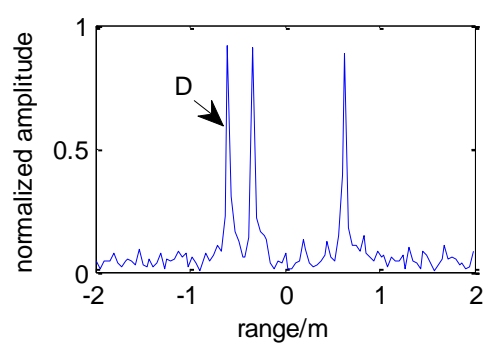

(a) radar 1

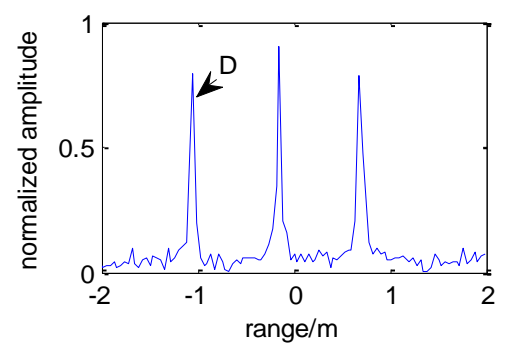

(b) radar 2

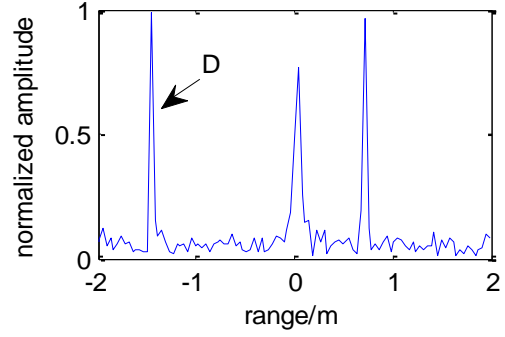

(c) radar 3

Fig. 4 The range profile sequence of the netted radar at certain time

Table 1 The range of three scattering points at some time of $\mathrm{m}$ radar

\begin{tabular}{ccccccc}
\hline$t$ & $0.100 \mathrm{~s}$ & $0.320 \mathrm{~s}$ & $0.436 \mathrm{~s}$ & $0.687 \mathrm{~s}$ & $0.830 \mathrm{~s}$ & $0.960 \mathrm{~s}$ \\
\hline Scattering point $s 1$ & -0.2250 & -0.5250 & -0.8625 & 0.0000 & -0.5250 & -0.825 \\
Scattering point $s 2$ & -0.4500 & -0.3750 & -0.3000 & -0.4875 & -0.3750 & -0.300 \\
Scattering point $s 3$ & 0.5438 & 0.6000 & 0.6375 & 0.4875 & 0.6000 & 0.6375 \\
\hline
\end{tabular}

Table 2 The estimation result of precession and structural parameters

\begin{tabular}{cccc}
\hline Parameters & Theoretical value & Estimated value & error \\
\hline$\theta / /^{\circ}$ & 10 & 9.8943 & $-1.06 \%$ \\
$h_{1} / \mathrm{m}$ & 2 & 1.9844 & $-0.78 \%$ \\
$h_{2} / \mathrm{m}$ & 0.5 & 0.5026 & $0.52 \%$ \\
$\mathrm{r} / \mathrm{m}$ & 0.5 & 0.5043 & $0.86 \%$ \\
$\alpha_{1}{ }^{\circ}$ & 100 & 98.95 & $-1.05 \%$ \\
$\alpha_{2} /^{\circ}$ & 115 & 116.187 & $0.99 \%$ \\
$\alpha_{3} /^{\circ}$ & 130 & 131.727 & $0.99 \%$ \\
\hline
\end{tabular}

\section{Conclusion}

Feature extraction of precession targets based on range-profile sequence has become the hot point of current research. But the research based on multi-aspect range-profile sequence in netted radar is not thorough. This text sets out from the extraction of precession feature and structure feature, making use of netted radar's multi view properties, finally successful extracts the precession parameters and parts of structure characteristic parameters. It breakthrough the limitation of single based radar could only offer the information in LOS and the researches did before only consider the ideal scattering center. It provides more accurate information for target's recognition.

\section{Acknowledgements}

This work was financially supported by the National Natural Science Foundation of China (63172166, 61501495). Corresponding author (E-mail: xudanlw@163.com) 


\section{References}

[1] Chen V C. Advances in application of radar micro-Doppler signatures[C].//Proc. of the 2014 IEEE Inf. on Antenna Measurements \& Application (CAMA), Antibes, France, 16-19 Nov, 2014, $1-4$.

[2] Ye C M, Xu J, Peng Y N, et al. Science China: Information Sciences, 2010, 53(8): 1641-1652.

[3] Teng Lei, Jinmang Liu, Fuping Yu, et al. Signal Processing, 2012, 28(1):73-79. (In Chinese)

[4] Xiaofeng Ai, Yongzhen Li, Feng Zhao, et al. Journal of Electronics \& Information Technology, 2011, 33(12): 2846-2851. (In Chinese)

[5] Hanying Yao, Wenfeng Sun, Xiaoyan Ma. Journal of Electronics \& Information Technology, 2013, 35(3): 537-544. (In Chinese)

[6] Sisan He, Huining Zhao, Cunqian Feng. Signal Processing, 2013, 29(8):1027-1035. (In Chinese)

[7] Xiaofeng Ai, Xiaohai Zou, Yongzhen Li, et al. Journal of Electronics \& Information Technology, 2011, 33(9): 2083-2088.(In Chinese)

[8] Li Xiao, Jianxiong Zhou, Jun He, et al. Acta Aeronautica ET Astronautica Sinica, 2010, 31(4): 812-818.(In Chinese)

[9] Xianda Zhang. Modern Signal Processing, fourth ed, Tsinghua University Press, Beijing 2002.(In Chinese) 